\title{
Accuracy assessment of pedicle screw insertion with patient specific 3D-printed guides through superimpose CT-analysis in thoracolumbar spinal deformity surgery
}

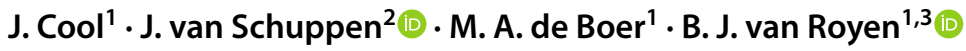

Received: 25 February 2021 / Revised: 6 July 2021 / Accepted: 28 July 2021 / Published online: 5 August 2021

(c) The Author(s) 2021

\begin{abstract}
Purpose In order to avoid pedicle screw misplacement in posterior spinal deformity surgery, patient specific 3D-printed guides can be used. An accuracy assessment of pedicle screw insertion can be obtained by superimposing CT-scan images from a preoperative plan over those of the postoperative result. The aim of this study is to report on the accuracy of drill guide assisted pedicle screw placement in thoracolumbar spinal deformity surgery by means of a superimpose CT-analysis. Methods Concomitant with the clinical introduction of a new technique for drill guide assisted pedicle screw placement, the accuracy of pedicle screw insertion was analyzed in the first patients treated with this technique by using superimpose CT-analysis. Deviation from the planned ideal intrapedicular screw trajectory was classified according to the Gertzbein scale. Results Superimpose CT-analysis of 99 pedicle screws in 5 patients was performed. The mean linear deviation was $0.92 \mathrm{~mm}$, the mean angular deviation was $2.92^{\circ}$ with respect to the preoperatively planned pedicle screw trajectories. According to the Gertzbein scale, $100 \%$ of screws were found to be positioned within the "safe zone".

Conclusion The evaluated patient specific 3D-printed guide technology was demonstrated to constitute a safe and accurate tool for precise pedicle screw insertion in spinal deformity surgeries. Superimpose CT-analysis showed a $100 \%$ accuracy of pedicle screw placement without any violation of the pedicle wall or other relevant structures. We recommend a superimpose CT-analysis for the first consecutive patients when introducing new technologies into daily clinical practice, such as intraoperative imaging, navigation or robotics.
\end{abstract}

Keywords 3D-printed guides $\cdot$ Pedicle screws $\cdot$ Superimpose CT-analysis $\cdot$ Spinal deformity surgery

\section{Introduction}

In spinal deformity surgery, the insertion of pedicle screws into the thoracic and lumbar vertebral bodies still makes for a challenging and technically demanding procedure [1]. Accurate pedicle screw insertion is of the utmost importance

\section{B. J. van Royen}

bj.vanroyen@amsterdamumc.nl

1 Department of Orthopedic Surgery, Amsterdam UMC, University of Amsterdam and Vrije Universiteit, Amsterdam Movement Sciences, Meibergdreef 9, 1105 AZ Amsterdam, The Netherlands

2 Department of Radiology and Nuclear Medicine, Amsterdam UMC, University of Amsterdam, Amsterdam Movement Sciences, Amsterdam, The Netherlands

3 Emma Children's Hospital, Amsterdam UMC, University of Amsterdam, Amsterdam, The Netherlands in order to prevent complications associated with pedicle screw misplacement.

Major structures that can be affected by pedicle screw misplacement include neuronal structures such as the spinal cord, major vessels such as the aorta or azygos vein, but also the trachea and pleurae, causing potentially life-threatening situations [2-4]. In freehand thoracolumbar pedicle screw placement using anatomical landmarks and intraoperative 2D fluoroscopy, pedicle screw misplacement is reported to be as high as 10-30\% according to postoperative CT imaging $[4,5]$. Consequently, there is a need for more accurate pedicle screw placement.

Many different intraoperative technologies to enhance pedicle screw insertion accuracy are currently available including imaging, navigation and robotics [6-9]. All of these innovative supporting technologies have been reported to demonstrate a high rate of accuracy for pedicle screw placement [6]. However, these options are also associated 
with various disadvantages including significant costs, extra time in surgery and additional radiation loads both for the patient as well as the surgical team. For intraoperative computer assisted navigation techniques, both a C-arm or CTscan and connected navigation system are required. These are reported to come at significant hospital costs for installation, intraoperative use as well as maintenance [10]. In addition, these fluoroscopy or CT-scan-based navigation systems expose the patient and surgical team to high radiation loads, which in turn increases the risk of developing associated cancers in long-term follow-up, both for the patient as well as the orthopedic surgeon [11-14].

More recently, the intraoperative use of patient specific 3D-printed guides for each vertebra to be instrumented has been reported to have a favorable effect on the accuracy of pedicle screw insertion [15-19]. For each instrumented vertebra, a 3D-printed guide is produced based on a preoperative protocolled low-dose CT-scan of the patient's spine. This technique, combined with digital preoperative surgical planning, has been proven to provide simple and accurate pedicle screw insertion planning both without the use of intraoperative C-arm fluoroscopy or CT guidance [15-19] and at significantly lower hospital costs. At the same time, when considering this as a new method to be adopted in clinical practice, a prospective risk analysis of potential adverse events is advisable. Our Hospital Quality and Risk \& Safety Department recommended to perform a postoperative assessment of pedicle screw placement accuracy for the first patients to be treated with the guidance this technique provides. Pedicle screw insertion accuracy assessment of the screws placed using this innovative method in spinal deformity surgery can be carried out by superimposing the CT-scan images used for preoperative planning over the postoperative CT-scan images of the inserted pedicle screws.

In this case series, we present the results of an accuracy assessment of pedicle screw insertion in thoracolumbar spinal deformity surgery, as assisted by patient specific 3D-printed guides, using superimpose CT-analysis of the first patients treated using this method in our institution.

\section{Methods}

\section{Patient population and preoperative planning}

We retrospectively evaluated the accuracy of pedicle screw insertion from prospectively collected patients. All patients, or their parents when under 18 years of age, gave us their written informed consent. The assessment of the accuracy of pedicle insertion was conducted for the first patients planned for spinal deformity surgery and treated by posterior spinal instrumentation of pedicle screws using 3D-printed patient specific guides in our institution from February 19th, 2020 to September 23rd, 2020.

For each case, a preoperative low-dose CT-scan of the patient's spine (slice thickness $0.6 \mathrm{~mm}$ ) was made and transformed into $3 \mathrm{D}$ reconstructions of each individual vertebra as well as the overall deformity. All scans were made using a 3rd generation dual-source CT-scanner (Somatom Force, Siemens Healthineers, Erlangen, Germany) at a tube potential of $100 \mathrm{kVp}$. For the first case, image acquisition was performed in accordance with the low-dose MySpine ${ }^{\circledR}$ CTscan protocol. This CT-scan protocol was developed further by the Radiology Department in our institution, resulting in an ultra-low dose (ULD) CT-scan protocol that was used for all ensuing patients. This adapted protocol was achieved by adding a built-in SPS (Selective Photon Shield) filter (Sn) in order to reduce the radiation dosage even further. All CT-scans were checked for appropriate quality. The resulting scans were encrypted and forwarded to the MySpine ${ }^{\circledR}$ Reconstruction \& Design Team (Medacta International SA, Switzerland). This team, using MySpine® proprietary computational software, would render the imagery into a 3D reconstruction of the patient's spinal deformity, including segmentation into each involved individual vertebra, prior to proposing a digital preoperative planning of pedicle screw entry points and ideal intrapedicular screw trajectories for each vertebral segment to be instrumented. After a required planning check, planning corrections as deemed fit and final validation - all by the appointed spine surgeon within an online web-based platform-the preoperative planning was finalized and automatically submitted for production of 3D-printed patient matching models and guides to be used prior to and during surgery. A 1:1 3D model of the total spinal deformity, 3D pilot hole drill guides and guide docking probes of each individual segment to be instrumented were printed (Fig. 1). These were subsequently forwarded to the hospital for final approval, surgical preparation and patient education. They were then autoclaved in the institution and presented for use during surgery, together with relevant instrument sets and sterile implants (MUST Pedicle Screw System, Medacta International SA, Switzerland). Guides and planned implants were grouped per individual segment so as to ease surgical flow.

\section{Surgical technique}

The standard protocol for spinal deformity surgery was used in all cases, including perioperative antibiotic prophylaxis, prone positioning on a spinal traction table and both intraoperative MEP (Motor Evoked Potential) and SSEP (Somatosensory Evoked Potential) monitoring. The high thoracic, thoracic and lumbar vertebrae to be instrumented were exposed using a posterior longitudinal midline incision. The anatomical landmarks of the spinous processes, 
Fig. 1 An example of a 3D printed vertebra and a matching patient specific 3D-printed guide



(a)



(b) laminae and facet joints were carefully prepared to ensure proper subsequent docking of the guides. Following anatomical identification of the mammillary process of T12 and the 12th rib, the 12th thoracic vertebral body was marked and confirmed by single shot PA and lateral 2D fluoroscopy image. With T12 as the starting point, the surgeon counted toward and identified the most cranial segment planned for pedicle screw instrumentation. Screw insertion was always started cranially in order to prevent subsequent potential interference from a caudally positioned screw with the next cranially positioned 3D guide. After meticulously checking the correct match between the patient, the guide and the segment to be addressed, the guide is carefully placed onto the posterior bony anatomy of the laminae. Correct docking of the proper $3 \mathrm{D}$ guides at the proper docking points as identified during preoperative planning was established by testing its stability. When in doubt, the guides were tested on the 1:1 model for reference. Once correctly in place, the intended pedicle screw entry point on the laminar cortex was prepared using a $2.7 \mathrm{~mm}$ high-speed burr through the first of the two 2.7-mm-pilot holes in the 3D-printed bilateral drill guide. This was done in order to prevent skiving of the subsequently used 2.7 -mm-drill bit with attached $30 \mathrm{~mm}$ positive stop, used to advance through the pilot hole in order to prepare the planned intrapedicular screw trajectory as determined by the relevant drill guide. A pedicle feeler was used to check for integrity of the pedicle walls and the cortex of the vertebral body, and a $2.7 \mathrm{~mm}$ temporary fixation pin was left in place through the guide in the first pilot hole before preparation of the contralateral screw trajectory was performed in identical fashion. After identical preparation and check of the second pilot hole and screw trajectory, bilateral calibrated blunt guide wires were placed. The 3D-printed guide was subsequently removed, making sure the wires were not displaced. The planned polyaxial, enhanced and cannulated pedicle screws (MUST PSS, Medacta International SA, Switzerland) were then implanted over the guide wires. All surgeries were performed by the same senior spine surgeon and fellow spine surgeon. No additional intraoperative $2 \mathrm{D}$ C-arm imagery was used, either for intraoperative screw placement or intraoperative and postoperative checking of ensuing screw positions.

\section{Postoperative evaluation of the screw position by superimpose analysis}

Postoperative evaluation of the pedicle screw entry point and screw trajectory was performed by superimpose analysis of the preoperative Low-Dose and ULD CT-scan images over a postoperative CT-scan using Solidworks® 2016 software. Any deviations of planned pedicle screw entry points and screw trajectories were assessed by comparing the planned screw positions with the actual screw positions using the preoperative and postoperative CT-scans. Accuracy was defined as the horizontal, vertical and depth deviation between the planned and postoperative screw entry point (in millimeters) and the sagittal and transversal deviation (in degrees of angulation). Additionally, the MySpine ${ }^{\circledR}$ proprietary software was used to visualize both the preoperatively planned positions and the actually achieved positions of the pedicle screws (Fig. 2).

\section{Postoperative screw positioning in the pedicle}

Pedicle screw accuracy was classified according to the Gertzbein scale [20] into four respective categories: grade 0 (screw completely in the pedicle), grade A (violation of the wall of the pedicle of less than $2 \mathrm{~mm}$ ), grade B (violation of 2-4 mm), grade $C$ (violation of more than $4 \mathrm{~mm}$ ). Screws classified as grade 0 or A were considered to be within the "safe zone" and thus correctly positioned, while grade B or $\mathrm{C}$ screws were considered to be mispositioned. The screws assessed "OUT" in accordance with the preoperative planning's intent, were not considered in the analysis.

\section{Statistical analysis}

For continuous data, the mean \pm standard deviation (SD) and range are given. IBM SPSS Statistics 26 (IBM Corp., Armonk NY) was used for statistical analysis. 
Fig. 2 Visualization of the superimposition analysis of T6 and $\mathrm{T} 7$ in case 4



\section{Results}

\section{Patient characteristics}

The first five patients (two male and three female) who underwent posterior spinal deformity correction surgery using MySpine ${ }^{\circledR}$ 3D-printed patient specific guides in our spine unit were analyzed. The mean patient age was $18.2 \pm 5.4(13-27)$ years. Two of those patients were treated for idiopathic scoliosis, two patients for syndromic/neuromuscular scoliosis, and one patient suffered from severe Scheuermann kyphosis. A total of 124 pedicle screws were planned, and 99 pedicle screws were inserted using the patient specific 3D-printed guides. For 8 of those pedicle screws, the preoperative planning noted an absence of adequate pedicles on the low-dose CT reconstruction, and these screws were therefore discarded (Figs. 3 and 4). In addition, the surgeon intraoperatively decided to not insert 17 contralateral pedicle screws in these five patients, despite those screws having been planned. Thus, a total of $79(80 \%)$ thoracic screws were placed, as well as $20(20 \%)$ lumbar screws. In most patients (except for the third), cranial 'topping-off' laminar hooks were placed so as to minimize the risk of proximal junctional kyphosis. None of the guided screws had to be inserted or replaced by freehand technique following iatrogenic damage to any of the defined docking points 
Fig. 3 The preoperative planning of bilateral pedicle screws in T4 in case 5. There is a small and flat pedicle on the right hand side on the low-dose CT reconstruction, and this screw was therefore discarded

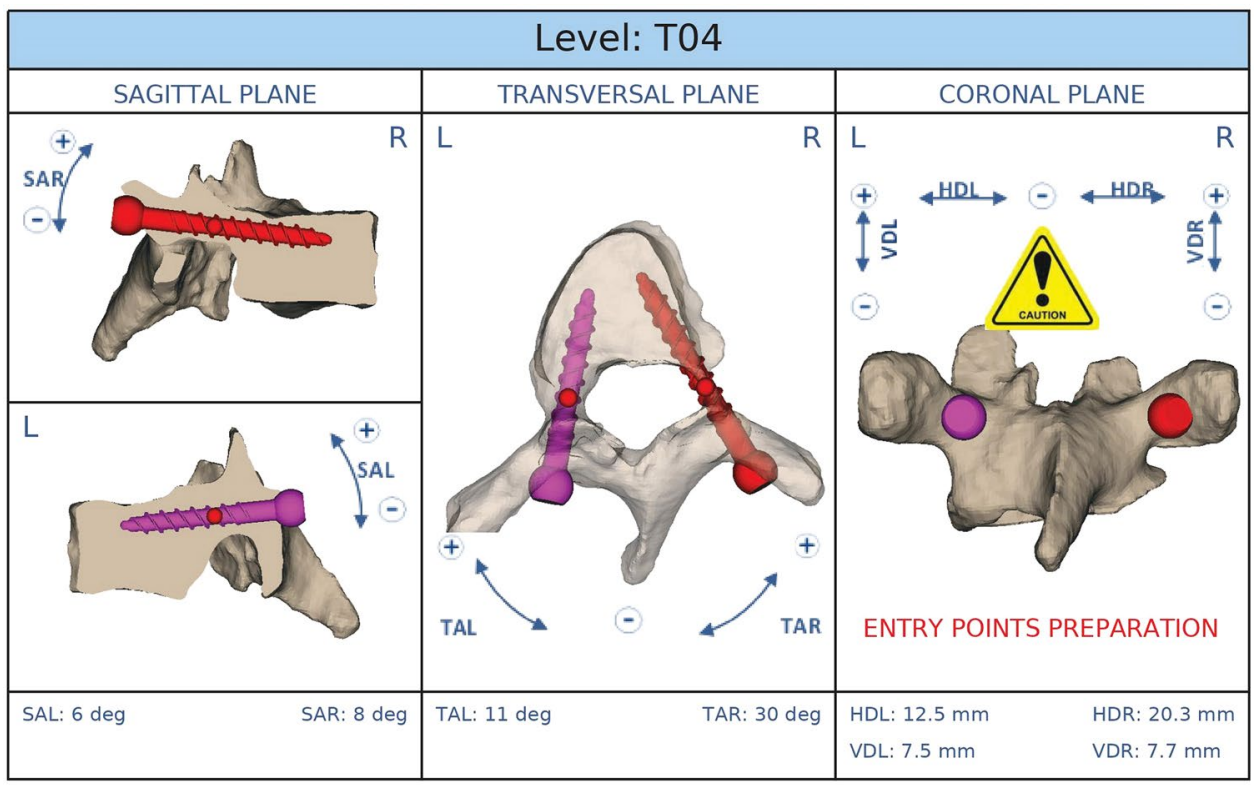

\begin{tabular}{|c|c|c|c|c|}
\hline $\begin{array}{c}\text { SCREW LENGTH } \\
\text { (cross-section in the screw plane) }\end{array}$ & 3D VIEW & $\begin{array}{c}\text { SCREW DIAMETER } \\
\text { (min cross-section of the pedicle) }\end{array}$ \\
\hline $\mathrm{L}$ & $\mathrm{R}$ & & \\
\hline $\begin{array}{c}\text { Length: } 30 \mathrm{~mm} \\
\text { Cortical gap: } 8 \mathrm{~mm}\end{array}$ & $\begin{array}{c}\text { Length: } 35 \mathrm{~mm} \\
\text { Cortical gap: } 8 \mathrm{~mm}\end{array}$ & & & \\
\hline
\end{tabular}

Fig. 4 Preoperative and postoperative PA and lateral radiographs of case 5

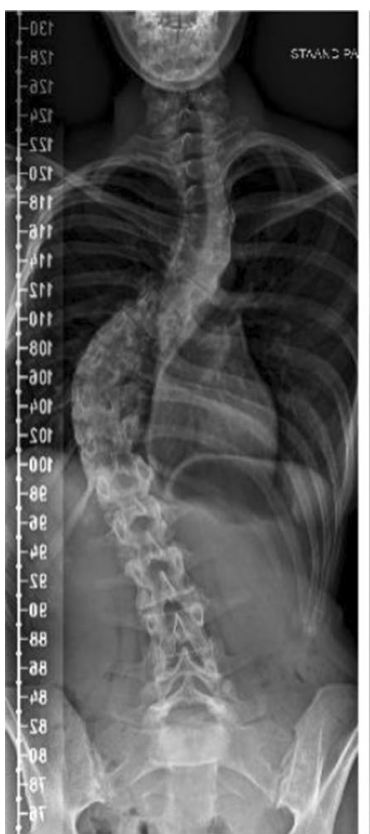

(a)

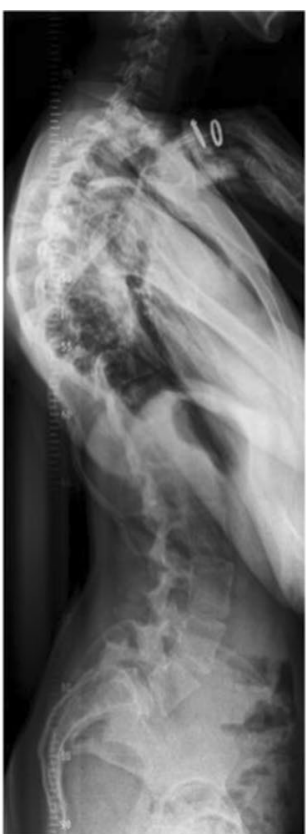

(b)

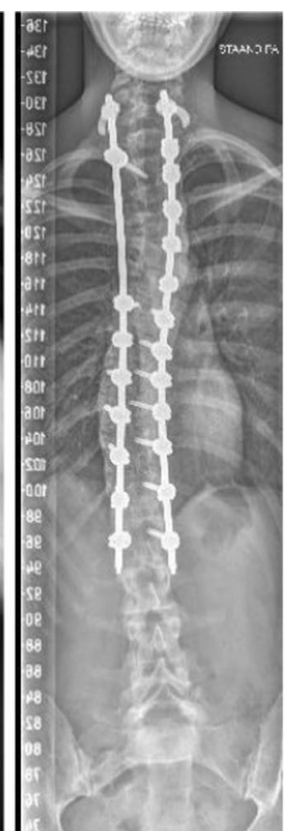

(c)



(d) 
on the instrumented vertebrae during dissection, resulting in inadequate or unstable positioning of the appropriate 3D-printed guide. There were no postoperative complications or surgical site infections. The patient characteristics are presented in Table 1.

\section{Postoperative evaluation of screw position}

A total of 99 pedicle screws were inserted using 3D-printed patient specific guides. Most of the analyzed screws (80\%) were placed in the high-thoracic and thoracic spine, and $100 \%$ of all screws were positioned within the safe zone, no more than 2-mm-outside of the pedicle. None of the screws perforated the cortex of the vertebral body. As mentioned before, none of the screws which were preoperatively planned to be partially OUT, were included in this analysis. The mean deviations between the planned pedicle screw entry point, depth and angular deviation of the placed pedicle screws evaluated by superimpose CT-analysis are given in Table 2: the mean vertical and horizontal deviation at the entry point were $1.03 \pm 0.0 .84$ (range $0.02-3.82$ ) $\mathrm{mm}$ and $0.81 \pm 0.9695$ (range $0.02-5.84$ ) $\mathrm{mm}$, respectively. The mean deviation in pedicle screw depth was $1.6665 \pm 1.25$ 24 (range 0.03-6.92) $\mathrm{mm}$, and the mean deviation in sagittal and axial angle was $3.2833 \pm 2.72^{\circ} 71^{\circ}$ (range $0.11-13.68$ ) and $2.5650 . \pm 0.2 .77^{\circ} 76^{\circ}$ (range $0-14.76$ ).

\section{Discussion}

Postoperative CT-analysis of pedicle screw insertion with 3D-printed guides in spinal deformity surgery showed a $100 \%$ accuracy of screw placement within the "safe zone" without any violation of the pedicle wall or other structures. Most inserted screws (80\%) were placed in the highthoracic and thoracic spine, and all analyzed thoracic and lumbar screws were positioned precisely within the pedicle. Superimpose CT-analysis demonstrated minimal deviations

Table 1 Patient characteristics. Radiation load of the preoperative Low-dose CT-scan measured for each patient in DLP (dose-length-product in $\mathrm{mGycm}$ ) and converted to the effective dose in $\mathrm{mSv}$ according to Deak [22]

\begin{tabular}{|c|c|c|c|c|c|}
\hline Patient & 1 & 2 & 3 & 4 & 5 \\
\hline Age (y) & 13 & 15 & 17 & 19 & 27 \\
\hline Gender & Female & Male & Female & Male & Female \\
\hline Diagnosis & $\begin{array}{l}\text { Idiopathic sco- } \\
\text { liosis (Lenke } \\
6 \mathrm{C})\end{array}$ & $\begin{array}{l}\text { Syndromic/ } \\
\text { Neuromuscular } \\
\text { scoliosis }\end{array}$ & $\begin{array}{l}\text { Syndromic/ } \\
\text { Neuromuscular } \\
\text { scoliosis }\end{array}$ & Scheuermann kyphosis & $\begin{array}{l}\text { Idiopathic } \\
\text { scoliosis } \\
\text { (Lenke 2B) }\end{array}$ \\
\hline $\begin{array}{l}\text { Planned instrumented vertebrae and screws } \\
\quad(n)\end{array}$ & T4-L4 (26) & T3-L3 (28) & T2-L1 (24) & T4-L1 (20) & T1-L1 (26) \\
\hline Number of placed pedicle screws $(n)$ & 21 & 20 & 20 & 18 & 20 \\
\hline Number of thoracic pedicle screws $(n)$ & 13 & 14 & 18 & 16 & 18 \\
\hline Number of lumbar pedicle screws $(n)$ & 8 & 6 & 2 & 2 & 2 \\
\hline Radiation exposure in DLP (mGycm) & 123 & 44 & 18 & 41 & 54 \\
\hline CT-scan length $(\mathrm{cm})$ & 26.6 & 54.3 & 42.9 & 69.5 & 64.3 \\
\hline Radiation exposure effective dose (mSv) & 2.21 & 0.74 & 0.26 & 0.53 & 0.70 \\
\hline
\end{tabular}

Table 2 The mean deviation between the planned pedicle screw entry point, pedicle screw depth and angular deviation of the placed pedicle screws of the 5 patients evaluated by superimpose CT-analysis

\begin{tabular}{llllllll}
\hline & & 1 & 2 & 3 & 4 & 5 & Mean \\
\hline$\Delta$ Vertical deviation (mm) @entry point & Mean (SD) & $0.69(0.38)$ & $1.86(1.04)$ & $0.86(0.74)$ & $0.78(0.61)$ & $0.79(0.68)$ & $1.03(0.84)$ \\
& Range & $0.02-1.27$ & $0.09-3.82$ & $0.04-2.41$ & $0.04-1.84$ & $0.08-2.43$ & $0.02-3.82$ \\
$\Delta$ Horiziontal deviation (mm) @entry point & Mean (SD) & $0.72(0.62)$ & $1.85(1.44)$ & $0.63(0.64)$ & $0.33(0.25)$ & $0.42(0.41)$ & $0.81(0.95)$ \\
& Range & $0.03-2.42$ & $0.14-5.84)$ & $0.04-2.09$ & $0.02-0.90$ & $0.05-1.70$ & $0.02-5.84$ \\
$\Delta$ Depth (mm) & Mean (SD) & $1.68(1.53)$ & $1.82(1.61)$ & $1.54(1.35)$ & $1.50(0.79)$ & $1.76(0.74)$ & $1.65(1.24)$ \\
& Range & $0.11-5.34$ & $0.15-6.92$ & $0.03-4.82$ & $0.47-3.80$ & $0.17-2.99$ & $0.03-6.92$ \\
$\Delta$ Sagittal angle $\left(^{\circ}\right)$ & Mean (SD) & $2.35(1.31)$ & $6.31(3.84)$ & $2.44(1.49)$ & $2.95(1.69)$ & $2.47(2.31)$ & $3.33(2.71)$ \\
$\Delta$ Transversal angle $\left({ }^{\circ}\right)$ & Range & $0.66-4.31$ & $0.89-13.68$ & $0.11-5.66$ & $0.41-5.86$ & $0.26-9.86$ & $0.11-13.68$ \\
& Mean (SD) & $2.00(1.33)$ & $3.64(4.28)$ & $2.45(2.90)$ & $1.83(1.52)$ & $1.48(1.33)$ & $2.50(2.76)$ \\
& Range & $0.44-5.16$ & $0.30-14.76$ & $0.00-11.43$ & $0.12-4.83$ & $0.08-5.35$ & $0.00-14.76$ \\
\hline
\end{tabular}


between planned and achieved pedicle screw positioning, at $<0.92 \mathrm{~mm}$ and $<2.91^{\circ}$, respectively, for linear and angular deviation. The achieved accuracy of pedicle screw entry point and intrapedicular screw trajectory fell well within the defined tolerances set for acceptance $(<1.5 \mathrm{~mm}$ for linear deviation and $<5^{\circ}$ for angular deviation). Most of the deviations were seen both at the insertion point and final positioning of the screw head and at the distal tip of the screw; not within the pedicle itself. The screw positions were possibly slightly altered during maneuvers associated with deformity correction [21]. Nevertheless, the absence of any pedicle wall violations or perforation of any vertebral body cortices, clearly demonstrates the high level of accuracy obtained while adopting this technique in our clinical practice.

The use of intraoperative patient specific 3D-printed guides for each instrumented vertebra in spinal deformity surgery renders the use of intraoperative 2D fluoroscopy for pedicle screw insertion superfluous. However, we still advise to intraoperatively check and identify the 12 th thoracic vertebral body by a single PA and lateral 2D fluoroscopy image and to subsequently count toward and determine the most cranial vertebra to be instrumented, prior to starting pedicle screw preparation and insertion. The use of intraoperative patient specific 3D-printed guides prevents the $\mathrm{C}$-arm from physically interfering with the surgical team during screw insertion. In addition, intraoperative radiation loads for both the patient and surgical team are minimized and in doing so the ALARA (as low as reasonably achievable) concept of radiation dosage is realized. On the other hand, a preoperative CT-scan is necessary in all cases for planning purposes. The radiation load of this preoperative low-dose CT-scan, however, is generally very modest, depending on the number of vertebrae selected for instrumentation, overall scanning length, the age of the patient, body mass index and gender. In our series, the dose-length-product (DLP) for the low-dose CT-scan according to the MySpine ${ }^{\circledR}$ protocol measured for the first case amounted to $123 \mathrm{mGycm}$. The mean DLP for the low-dose CT-scan according to the local ULD MySpine ${ }^{\circledR}$ protocol with an additional SPS filter (Sn), amounted to $39,25 \mathrm{mGycm}$ (range 18-54) in the next 4 patients (Table 1). The effective dose, calculated by using the conversion factor for DLP to effective dose, adapted for age and body part and according to the new International Commission on Radiological Protection (ICRP) recommendations [22], was $2.21 \mathrm{mSv}$ in the first patient. The mean effective dose for the next 4 patients amounted to $0.56 \mathrm{mSv}$ (range 0.26-0.74) (Table 1). In comparison, the mean effective dose of a standard standing full spine PA and lateral radiograph is $0.7 \mathrm{mSv}$ [14].

Of the planned 124 screws, a total of 8 screws could not be inserted because of the flat anatomy of the pedicles, as revealed on the low-dose CT-scan (Fig. 3). In our experience, we found that the 3D digital preoperative planning helps the surgeon to recognize these occasionally flat pedicles, often located at the concave side of the thoracic curvature, and thereby helps preventing accidental neurological complications potentially caused by screw insertion attempts into these altered pedicles. In a total number of 17 instances, the surgeon intraoperatively decided not to insert a contralateral screw so as to both avoid a $100 \%$ screw density as well as facilitate posterior spinal fusion.

Correct docking of the 3D guide requires meticulous preparation of the bony anatomy including the spinous process. However, removing the interspinous ligament at the most proximal instrumented thoracic vertebra possibly increases the risk of postoperative proximal junctional kyphosis. In order to prevent future proximal junctional kyphosis in thoracolumbar spinal deformity surgery, we advise adding proximal 'topping-off' laminar hooks into the instrumentation.

Before introducing this new patient matching technique for pedicle screw insertion in spinal deformity surgery with intraoperative patient specific 3D-printed guides into our clinical practice, a prospective risk analysis was performed, according to the Bow-Tie model [23]. This was done in order to identify existing and missing barriers for potential critical events. The analysis was carried out by a multidisciplinary team, consisting of two orthopedic surgeons, the unit head of the OR department, a scrub nurse, a representative of the central sterilization department, a representative of the radiology department, an independent neurosurgeon and a representative of the company providing us with the technology and hardware. The analysis was supervised by 2 senior advisors of the Hospital Quality and Risk \& Safety department. The analyzing team defined the potential critical events in a brainstorming session on how to avoid the patients suffering from any potential adverse event associated with the clinical adoption of a new surgical technique. Subsequently, the risk factors and the current and required barriers in the pre, per- and postoperative process were identified. Based on the reported outcome of this meeting, an implementation plan with defined responsibilities and associated timelines was developed. In total, 18 potential adverse events were identified, analyzed and addressed in this plan. In addition, some critical features regarding the intended use of 3D-printed patient specific guides in spinal deformity cases were recognized. First of all, at the time of preforming this prospective risk analysis, it routinely took the supplying company two to three weeks to have all components of the technology available for elective surgery. This technique, therefore, is not suitable for use in acute procedures such as trauma or oncology cases. Spinal deformity surgeries however, are planned well in advance, which makes the use of 3D-printed patient specific guides a viable option for this type of surgery. Secondly, since confidential patient information is, albeit encrypted, shared digitally with the team from MySpine ${ }^{\circledR}$, 
provisions and requirements related to processing and saving of patient's data according to the General Data Protection Regulation (GDPR) should be taken into account. Finally, screw misplacement was considered to constitute a significant potential adverse event to be measured and evaluated. It was therefore recommended to include a postoperative assessment of screw insertion accuracy in the first 5 patients as part of the implementation plan. This recommendation served as the basis for the current report.

We acknowledge several limitations to this study. Firstly, this was a single-center study involving five consecutive patients. For a more reliable superimpose CT-analysis of pedicle screw insertion accuracy using 3D-printed patient specific guides for spinal deformity surgeries, a larger number of evaluated patients and pedicle screws would be needed. We did, however, demonstrate a high accuracy of pedicle screw insertion in the first 5 patients we treated with the help of this technology, while routinely planned postoperative CT-scan imaging for such an assessment would significantly increase the cumulative radiation loads of these patients. Secondly, these five consecutive patients were all operated on by a single surgical team. This might have had an effect on the results based on the nature of its learning curve. Thirdly, we did not report on clinical patient outcomes such as fixation failure, quality of life assessments or fusion rates, which would have emanated from a long-term follow-up. These should also be evaluated in future studies.

\section{Conclusion}

In this case series, pedicle screw insertion aided by patient matching technology and patient specific 3D-printed guides in the surgical correction of spinal deformities showed a $100 \%$ accuracy of screw placement without any violations of the pedicle wall or other important structures and a trajectory accuracy with mean linear and angular deviations of $<0.92 \mathrm{~mm}$ and $<2.91^{\circ}$, respectively. Superimpose CTanalysis appears to be a useful method to analyse and visualize the accuracy of pedicle screw insertion when adopting the abovementioned technology in daily clinical practice. We recommend this method of analysis as a quality control for the first consecutive patients during the implementation of any new intraoperative imaging, navigation or robotics technology introduced into daily clinical practice.

Acknowledgements We thank N.H.J. Lobe, radiologic technologist, for his work on the adapted ultra-low dose (ULD) MySpine ${ }^{\circledR}$ protocol by adding an SPS on the tube voltages.

Author contribution All authors contributed to the study conception and design. Material preparation, data collection and analysis were performed by JC, JS, MAB and BJR. The first draft of the manuscript was written by JC, and all authors commented on previous versions of the manuscript. All authors read and approved the final manuscript.

Funding Not applicable. No funding was received for conducting this study.

\section{Declarations}

Conflict of interest The authors certify that they have no affiliations with or involvement in any organization or entity with any financial interest (such as honoraria; educational grants; participation in speakers' bureaus; membership, employment, consultancies, stock ownership or other equity interest; and expert testimony or patent-licensing arrangements) or non- financial interest (such as personal or professional relationships, affiliations, knowledge or beliefs) in the subject matter or materials discussed in this manuscript.

Consent for publication Patients and/or parents provided informed consent regarding publishing their data and photographs.

Ethical approval Ethical approval was waived by the local Ethics Committee of Amsterdam UMC, University of Amsterdam in view of the retrospective nature of the study, and all the procedures being performed were part of the routine care (reference number W21_090 \# 21.101)

Availability of data and material The datasets generated during and/or analyzed during the current study are available from the corresponding author on reasonable request.

Consent to participate Informed consent was obtained from all individual participants and/or parents included in the study.

Informed Consent Additional informed consent was obtained from all individual participants for whom identifying information is included in this article.

Open Access This article is licensed under a Creative Commons Attribution 4.0 International License, which permits use, sharing, adaptation, distribution and reproduction in any medium or format, as long as you give appropriate credit to the original author(s) and the source, provide a link to the Creative Commons licence, and indicate if changes were made. The images or other third party material in this article are included in the article's Creative Commons licence, unless indicated otherwise in a credit line to the material. If material is not included in the article's Creative Commons licence and your intended use is not permitted by statutory regulation or exceeds the permitted use, you will need to obtain permission directly from the copyright holder. To view a copy of this licence, visit http://creativecommons.org/licenses/by/4.0/.

\section{References}

1. Avila MJ, Baaj AA (2016) Freehand thoracic pedicle screw placement: review of existing strategies and a step-by-step guide using uniform landmarks for all levels. Cureus 8:e501. https://doi.org/ 10.7759/cureus.501

2. Flynn JM, Sakai DS (2012) Improving safety in spinal deformity surgery: advances in navigation and neurologic monitoring. Eur Spine J 22:131-137. https://doi.org/10.1007/s00586-012-2360-6 
3. Wegener B, Birkenmaier C, Fottner A, Jansson V, Dürr HR (2008) Delayed perforation of the aorta by a thoracic pedicle screw. Eur Spine J 17:351-354. https://doi.org/10.1007/s00586-008-0715-9

4. Sarlak AY, Tosun B, Atmaca H, Sarisoy HT, Buluç L (2009) Evaluation of thoracic pedicle screw placement in adolescent idiopathic scoliosis. Eur Spine J 18:1892-1897. https://doi.org/10. 1007/s00586-009-1065-y

5. Etemadifar M, Jamalaldini M (2017) Evaluating accuracy of freehand pedicle screw insertion in adolescent idiopathic scoliosis using postoperative multi-slice computed tomography scan. Adv Biomed Res 6:19. https://doi.org/10.4103/2277-9175.201331

6. Gelalis ID, Paschos NK, Pakos EE, Politis AN, Arnaoutoglou CM, Karageorgos AC, Ploumis A, Xenakis TA (2012) Accuracy of pedicle screw placement: a systematic review of prospective in vivo studies comparing free hand, fluoroscopy guidance and navigation techniques. Eua Spine J 21:247-255. https://doi.org/ 10.1007/s00586-011-2011-3

7. Tormenti MJ, Kostov DB, Gardner PA, Kanter AS, Spiro RM, Okonkwo DO (2010) Intraoperative computed tomography imageguided navigation for posterior thoracolumbar spinal instrumentation in spinal deformity surgery. Neurosurg Focus 28:E11. https:// doi.org/10.3171/2010.1.Focus09275

8. Siddiqui MI, Wallace DJ, Salazar LM, Vardiman AB (2019) Robot-assisted pedicle screw placement: learning curve experience. World Neurosurg 130:e417-e422. https://doi.org/10.1016/j. wneu.2019.06.107

9. Flynn JM, Sakai DS (2013) Improving safety in spinal deformity surgery: advances in navigation and neurologic monitoring. Eur Spine J 22(Suppl 2):S131-137. https://doi.org/10.1007/ s00586-012-2360-6

10. Malham GM, Wells-Quinn T (2019) What should my hospital buy next?-Guidelines for the acquisition and application of imaging, navigation, and robotics for spine surgery. J Spine Surg 5:155165. https://doi.org/10.21037/jss.2019.02.04

11. Simony A, Hansen EJ, Christensen SB, Carreon LY, Andersen MO (2016) Incidence of cancer in adolescent idiopathic scoliosis patients treated 25 years previously. Eur Spine J 25:3366-3370. https://doi.org/10.1007/s00586-016-4747-2

12. Valone LC, Chambers M, Lattanza L, James MA (2016) Breast radiation exposure in female orthopaedic surgeons. J Bone Jt Surg Am 98:1808-1813. https://doi.org/10.2106/jbjs.15.01167

13. Pitteloud N, Gamulin A, Barea C, Damet J, Racloz G, Sans-Merce M (2017) Radiation exposure using the O-arm(®) surgical imaging system. Eur Spine J 26:651-657. https://doi.org/10.1007/ s00586-016-4773-0

14. Law M, Ma WK, Lau D, Chan E, Yip L, Lam W (2016) Cumulative radiation exposure and associated cancer risk estimates for scoliosis patients: Impact of repetitive full spine radiography. Eur J Radiol 85:625-628. https://doi.org/10.1016/j.ejrad.2015.12.032

15. Cecchinato R, Berjano P, Zerbi A, Damilano M, Redaelli A, Lamartina C (2019) Pedicle screw insertion with patient-specific 3D-printed guides based on low-dose CT-scan is more accurate than free-hand technique in spine deformity patients: a prospective, randomized clinical trial. Eur Spine J 28:1712-1723. https:// doi.org/10.1007/s00586-019-05978-3

16. Farshad M, Betz M, Farshad-Amacker NA, Moser M (2017) Accuracy of patient-specific template-guided vs. free-hand fluoroscopically controlled pedicle screw placement in the thoracic and lumbar spine: a randomized cadaveric study. Eur Spine J 26:738-749. https://doi.org/10.1007/s00586-016-4728-5

17. Matsukawa K, Kaito T, Abe Y (2020) Accuracy of cortical bone trajectory screw placement using patient-specific template guide system. Neurosurg Rev 43:1135-1142. https://doi.org/10.1007/ s10143-019-01140-1

18. Lamartina C, Cecchinato R, Fekete Z, Lipari A, Fiechter M, Berjano $P$ (2015) Pedicle screw placement accuracy in thoracic and lumbar spinal surgery with a patient-matched targeting guide: a cadaveric study. Eur Spine J 24(Suppl 7):937-941. https://doi.org/ 10.1007/s00586-015-4261-y

19. Kaito T, Matsukawa K, Abe Y, Fiechter M, Zhu X, Fantigrossi A (2018) Cortical pedicle screw placement in lumbar spinal surgery with a patient-matched targeting guide: a cadaveric study. $\mathrm{J}$ Orthop Sci 23:865-869. https://doi.org/10.1016/j.jos.2018.06.005

20. Gertzbein SD, Robbins SE (1990) Accuracy of pedicular screw placement in vivo. Spine 15:11-14. https://doi.org/10.1097/00007 632-199001000-00004

21. Senkoylu A, Cetinkaya M (2017) Correction manoeuvres in the surgical treatment of spinal deformities. EFORT Open Rev 2:135140. https://doi.org/10.1302/2058-5241.2.170002

22. Deak PD, Smal Y, Kalender WA (2010) Multisection CT protocols: sex- and age-specific conversion factors used to determine effective dose from dose-length product. Radiology 257:158-166. https://doi.org/10.1148/radiol.10100047

23. Kerckhoffs MC, van der Sluijs AF, Binnekade JM, Dongelmans DA (2013) Improving patient safety in the ICU by prospective identification of missing safety barriers using the bow-tie prospective risk analysis model. J Patient Saf 9:154-159. https://doi.org/ 10.1097/PTS.0b013e318288a476

Publisher's Note Springer Nature remains neutral with regard to jurisdictional claims in published maps and institutional affiliations. 